\title{
Réflexion, réflexivité et acquisition des langues
}

\author{
Anne TREVISE \\ Université de Paris X - Nanterre
}

\section{Présentation}

Les différents articles de ce volume présentent un ensemble très varié de réflexions sur les activités et les représentations métalinguistiques d'enfants et d'adultes, au cours de l'acquisition de la langue "maternelle" ou d'une langue étrangère, en milieu scolaire et en milieu dit "social" ou "naturel".

L'article de J.-E. Gombert traite du développement des activités métalinguistiques et des connaissances sur le langage de l'enfant monolingue.

M.-T. Vasseur et J. Arditty recensent quinze ans de recherches sur les activités réflexives en situation de communication exolingue et sur leurs rôles dans l'acquisition des langues 2 .

D. Huot et R. Schmidt procèdent d'abord à une mise au point des terminologies existantes en Amérique du Nord et en France dans les recherches sur la conscience et l'activité métalinguistiques. Puis, ils présentent brièvement trois études longitudinales portant sur l'acquisition de différentes langues 2 par deux adultes et une enfant de langues maternelles diverses.

S. Haller et B. Schneuwly rendent compte des activités méta- et épilinguistiques lors de la production à trois adultes d'un texte argumentatif écrit en français langue étrangère, en milieu scolaire.

F. François clôt ce volume en nous offrant une réflexion sur la genèse du fonctionnement langagier, alimentée d'exemples sur la nature des discours enfantins qui se reprennent et se modifient les uns les autres.

Les mêmes questions parcourent toutes ces réflexions, "états de la question", et présentations de cas, même si les approches théoriques sont parfois différentes. La 
convergence de la majorité des préoccupations se fait sur des études des fonctionnements des différents états de langues et des phénomènes de réflexivité en interaction.

Mon propre travail introductif ici n'ira donc pas vers un nouvel "état de la question", ou des différentes questions, mais, à partir de ces angles d'approche du langage que sont les différentes situations d'acquisition, il s'attachera à démêler quelques uns des fils qui tissent les liens entre langage et réflexivité, entre représentations et activités métalinguistiques, et entre ces dernières et l'acquisition. 


\section{Clairement énoncer pour bien concevoir}

Mais les mots pour le dire ne viennent pas si aisément...

On répète souvent que la métalangue est dans la langue. Elle n'échappe pas aux contraintes de négociations du sens et d'ajustements divers qui caractérisent le fonctionnement d'une langue. Une réflexion sur le sens, ou les sens, à apporter aux mots métalangue, métalangage, métalinguistique, métadiscours, activités métalinguistiques ou épilinguistiques, représentations métalinguistiques, montre que si l'on veut communiquer, il faut s'entendre un minimum sur la définition que l'on donne aux mots, et comme souvent, on s'aperçoit qu'une réflexion en vue d'une clarification terminologique impose une redéfinition de la pensée. Benveniste écrivait que "c'est ce qu'on peut dire qui délimite et organise ce qu'on peut penser. La langue fournit la configuration fondamentale des propriétés reconnues par l'esprit aux choses" (1966 : 70). L'étiquetage dirige la pensée plus que l'inverse. Une étude sur les métadiscours, leurs objets, les tissages plus ou moins serrés des activités langagières et métalangagières, et leurs rôles dans les acquisitions des langues (premières ou secondes), se doit d'utiliser un métalexique aussi cohérent et clair que possible, sous peine de dissolution progressive du champ d'investigation.

Les métatermes utilisés ici réfèrent à des notions aux contours parfois labiles. Ce ne sont pas des concepts munis d'une définition stricte, immuable, autonome (Grize, 1995 : 48-49). Notre discours utilise une langue naturelle, avec tous les aléas de celleci, mais elle a aussi un dynamisme propre et une possibilité d'évolution.

Grize explique :

un certain flou a l'avantage d'ouvrir la signification à des sens multiples, condition sine qua non du progrès de la pensée. De schématisations en schématisations, les notions se transforment et elles évoluent, ce que ne font jamais les concepts. (...) Un concept ne se modifie jamais dans la langue-objet. Il faut pour cela que la pensée change de plan et se serve d'une métalangue, de sorte que seules les notions ont un réel devenir au sein de la langue qui les présente. (Ibid. : 51)

Cette réflexion vaut, me semble-t-il, pour nos recherches et tentatives de clarification de ces champs d'investigation que sont la réflexivité inhérente au langage et les différentes activités et représentations métalinguistiques. Je ne prétendrai donc pas définir ou redéfinir des concepts, mais j'évaluerai la pertinence de certaines notions, en les explicitant autant que faire se peut, pour ce moment de nos recherches.

Il n'y a pas de raison que les métadiscours échappent aux contraintes de l'élasticité du langage, et à la nature dynamique du sens des mots : le mot cheval va changer de sens suivant les contextes, ou le ton ironique, et un dictionnaire ne peut que énumérer 
les usages divers du mot, ses sens figurés, mais ne peut prévoir tous les usages métaphoriques, métonymiques et autres déplacements opérés par les sujets parlants, sans mentionner les jeux de mots du type chval dire à ma mère (pour expliquer que le cheval est l'animal le plus rapporteur). Les mots conscience, représentation, activité, ou pensée, pour prendre ceux qui posent sans doute le plus de problèmes définitionnels, ouvrent certes des abîmes d'interprétations, mais ces foisonnements stimulent aussi ladite pensée.

Nous garderons toujours ceci en mémoire, car l'élasticité et, précisément, la réflexivité inhérentes au langage permettent ce jeu dans la mécanique, et donc les jeux. Les termes métalinguistiques se parent souvent de sérieux et de scientificité, mais n'échappent pas pour autant à ces contraintes, qui sont d'ailleurs une telle source d'intérêt, voire de jouissance...

\section{Langage et réflexivité}

\subsection{Dichotomies et schématismes heuristiques}

Dans le fonctionnement de la pensée, il est nécessaire de distinguer les représentations ou contenus de pensée, et l'activité même de penser.

De manière heuristique, il est utile, par ailleurs, de procéder à deux autres dichotomies, quitte à les récuser ensuite, ou du moins à en cerner les schématismes.

La première dichotomie se propose de distinguer :

- les discours métalinguistiques, constitués, observables, qui ont clairement pour objets à la fois les codes linguistiques, la communication, les représentations sur les langues, l'apprentissage. Ces productions métalinguistiques émanent de linguistes, d'enseignants, d'apprenants de L1 ou de L2, et instituent clairement une objectivation consciente des phénomènes linguistiques divers dont ils traitent. On a là des contenus de pensée, des représentations métalinguistiques. On pourrait parler ici d'une verticalité, et de métadiscours externes, pour aller vite ;

- "le métalinguistique", substantif que j'emploierai ici pour renvoyer à toutes les traces observables de la réflexivité constitutive des activités langagières. Il s'agit des phénomènes très hétérogènes qui témoignent de prises de "distance", pour utiliser une métaphore psychologisante, de changements de plans divers effectués par les locuteurs par rapport à leurs dires et aux dires de l'autre dans l'interaction. Ces phénomènes peuvent englober gestes, sourires et mimiques, mais nous nous "contenterons" de considérer ici les traces verbales observables. A propos de ce métalinguistique beaucoup plus interne à l'activité langagière, tissé par et avec elle, se pose le problème des degrés de conscience. Ces marques du métalinguistique sont les traces de l'activité de langage elle-même issues de représentations plus ou moins conscientes. 
La deuxième dichotomie sépare :

- ces phénomènes observables en tant que traces dans les productions des sujets, qu'ils relèvent de métadiscours ou du métalinguistique ;

- et tout ce qui est du domaine de l'inobservable, de l'éventuellement inférable, que je nommerai très grossièrement représentations et activités métalinguistiques. Ces activités, qui relèvent de degrés de conscience divers, "accompagnent" (parfois), notamment dans le cours de l'acquisition, les processus de compréhension, de production, de références à des règles de diverses natures, à des représentations métalinguistiques, contrastives ou non. On parle ici d'activités métacognitives, planifiées et organisées par des métadiscours intérieurs, en liaison avec des contenus de pensée, des représentations. On reste dans le domaine de ce dont on pense que les sujets ont conscience. Ces inobservables de l'acquisition et des pratiques d'interaction, peuvent parfois être inférés, soit, avec prudence, des conduites linguistiques, des hésitations ou reprises dans des productions, des malentendus avérés en compréhension, des questionnements dans les cas d'incompréhension révélée, soit, avec encore plus de prudence, des verbalisations métalinguistiques des sujets. Ces verbalisations métalinguistiques, spontanées ou élicitées, sont à prendre comme les traductions, les reconstructions verbales, plus ou moins fidèles des représentations métalinguistiques conscientes. Elles peuvent être produites par des sujets à propos de leurs propres conduites ou productions, ou à propos d'autres productions auxquelles ils sont confrontés, en langue 1 ou en langue 2. Mais elles ne préjugent guère des activités métalinguistiques qui auraient ou non accompagné leurs propres conduites linguistiques de production ou de reconnaissance, ni de leurs rôles éventuels dans les processus langagiers, acquisitionnels ou non. Elles préjugent encore moins, on le sait, des activités cognitives non conscientes de production et de reconnaissance, de mémorisation ou d'automaticité, ou des mouvements cognitifs de passage de la conscience à l'automatisation, et de "retours" à la conscience en cas d'obstacle.

\subsection{Métadiscours des linguistes, autres métadiscours et métalinguistique}

Les termes "métalangue" et "métalangage" me semblent souvent mal définis, injustifiés, ou insuffisants.

On sait que Jakobson (1963 : 53-54) a parlé de la distinction entre langage-objet et métalangage, et de sa pertinence non seulement pour les linguistes, mais aussi pour décrire les "activités linguistiques usuelles" de négociations de sens en interaction. Il insistait sur la nécessité du recours au métalangage pour l'acquisition du langage et pour son fonctionnement normal, et sur les pertes du métalangage dans les carences 
aphasiques. Benveniste parlait lui, non de métalangage, mais de métalangue, comme d'une langue dont la seule et unique fonction est de décrire une langue (1974: 35).

Culioli, quant à lui, écrivait :

On peut se placer du point de vue du sujet-énonciateur-locuteur qui a une activité métalinguistique non consciente (je parle alors d'activité épilinguistique) ou qui, par les jeux de langage de tous ordres, s'adonne à la jouissance du métalinguistique. A son propos, on pourrait soutenir qu'il y a du métalinguistique, mais pas de métalangage, au sens d'un langage extérieur à la langue-objet. (...) (le) linguiste (...) construit un système de représentation métalinguistique afin de pouvoir (1) représenter les phénomènes grâce à des représentants ayant des propriétés formelles (...), (2) calculer. (...)

Rien ne dit d'ailleurs que notre activité cognitive et notre activité épilinguistique soient correctement représentées par notre système de représentation métalinguistique, au sens réaliste de "correctement", mais nous sommes amenés à poser que les calculs renvoient à des opérations, les simulent, sans plus. Y a-t-il plusieurs sortes de métalinguistique ? Je serais tenté de répondre "oui", entre la glose (jugement d'équivalence), les reprises, les ré-analyses ("quand je pose la question "Est-ce que tu viendras ?", ça veut dire que je veux savoir si tu viendras ou non"), les jugements d'acceptabilité, les représentations à base métaphorique (par exemple dans les phénomènes portant sur la temporalité), les représentations de l'ordre du topologique, etc., il va (...) y avoir du métalinguistique et non une métalangue. (1990: 41)

Par ailleurs Culioli écrivait aussi :

Le plus souvent, il faut le reconnaître, nous ne prenons pas nos distances par rapport au langage ; (...) nous nous le sommes tellement approprié qu'il semble être devenu un simple instrument docile qui obéit à nos intentions (...). Fascinés par le sens, (...) nous n'arrivons pas à prendre au sérieux la forme, nous confondons aisément (...) la réalité extralinguistique et l'outil linguistique (...). Il arrive que le langage soit remis en question, qu'il ne soit plus aussi transparent que nous l'imaginons parfois ; un malentendu peut nous révéler l'ambiguïté foncière des langues naturelles : les mots, ces médiateurs par excellence, nous font ressentir leur opacité, et nous révèlent qu'il n'y a pas une relation immédiate entre les mots et les choses. (1967:65-73)

Tant Jakobson que Culioli distinguent entre la métalangue du linguiste et le métalinguistique dans l'utilisation quotidienne, usuelle, du langage.

\section{Le métadiscours des linguistes}

Le linguiste travaille donc à construire un système de représentation qui porte sur ce système de représentation qu'est une langue, pour reprendre les termes de Culioli (1990 : 21). Son discours métalinguistique sera cohérent, utilisera des concepts définis aussi scientifiquement ou techniquement que possible, et les métaphores seront justifiées et explicitées. Néanmoins, les silences métalinguistiques sont grands : seules 
certaines zones sont modélisées. La linguistique ne rend pas (encore) compte de tout, et il reste toujours l'individualité du sujet humain. C'est pourquoi Damourette et Pichon ont pu parler de la grammaire "en tant que mode d'exploration de l'inconscient"1. Elle devait d'ailleurs, pour eux, être élaborée par des grammairiens pour qui la langue décrite devait être leur langue maternelle.

Culioli insiste par ailleurs sur le fait qu'il n'est pas du tout certain que ces représentations métalinguistiques représentent l'activité cognitive non consciente des sujets : il s'agit d'une simulation d'opérations cognitives, de simulations du savoir épilinguistique (défini comme du savoir métalinguistique non conscient) des locuteurs, dont par définition ils n'ont pas conscience en parlant ou en comprenant.

Il est donc nécessaire de ne pas assimiler règles du produit et règles de production, et de ne pas prétendre à une quelconque réalité psychologique des règles dégagées. Le "coup de force philosophique" de Chomsky, pour reprendre l'expression d'Auroux (1996 : 311), a été de dire que les règles algorithmiques sont non seulement des lois que doit découvrir et formuler le linguiste, mais qu'elles ont aussi les moyens qui, implémentés dans le cerveau des hommes, permettent à ceux-ci de parler. Cette simulation du comportement humain est aussi ce que tentent les connexionnistes à l'heure actuelle. Cette psycholinguistique est loin des attitudes descriptivistes des linguistes positivistes du siècle dernier.

Néanmoins, hors de l'abstraction des concepts métalinguistiques, nul ne pourrait songer à dégager des invariants linguistiques et cognitifs (Desclés, 1994). Ce mouvement du particulier vers le générique, le généralisable, et le mouvement inverse sont des conduites universelles qui ne s'appliquent pas seulement à l'observation du langage, mais à toute notre perception du monde.

Là se jouent la pertinence et l'objectif de la représentation métalinguistique : si elle n'est pas adéquate, si elle s'enferme dans des concepts qui n'épousent pas la richesse et le dynamisme du langage dans son fonctionnement, elle peut n'être qu'un piètre écran, et non un outil de découverte de ce fonctionnement spécifiquement humain. Seule une métalangue adéquate qui théorise à partir de corpus suffisamment larges, n'occultant pas les observables, permet de percevoir l'objet avec clarté, quitte à ce que ses étiquettes et ses métaphores soient réajustées au fil de la recherche.

Pour prendre un exemple, si on s'arrête à la caractérisation de voiture comme nom dénombrable, on ne va pas pouvoir rendre compte du dynamisme des fonctionnements dans l'énoncé Ya de la voiture aujourd'hui. Il est certainement plus intéressant de considérer les phénomènes nominaux ou verbaux, sur un plan plus abstrait et plus

\footnotetext{
1 Titre d'un article des auteurs, paru en 1925, et cité par Arrivé, $1994: 144$.
} 
généralisable, en termes de qualitatif ou de quantitatif (de Vogüé, 1989, Franckel et Paillard, 1992, Trévise, 1995). Les métadiscours descriptifs construisent leur objet à partir des observables linguistiques, qui sont toujours interprétés par le regard théorique. On sait que les données sont forcément du construit : elles ne peuvent être observables que dans un va-et-vient avec une théorie de l'observation, et donc une métalangue d'observation qui les catégorise, en fait ressortir les aspects discriminants.

\section{Les autres types de métadiscours}

Les métadiscours du linguiste ne sont pas de la même teneur que ceux de l'historien d'une langue, du philosophe du langage, de l'enseignant de langue, ou des manuels (L1 ou L2), ou encore ceux de l'apprenant. Se manifestent là différentes formes de labilités sémantiques des métatermes utilisés avec différents degrés de sophistication. Le discours de l'enseignant est médiatisé, et on note souvent la différence entre le savoir dit "savant" et le savoir enseigné, avec les heurs et malheurs que l'on connaît, et qu'il est nécessaire, et facile, de vilipender.

Les métatermes auront encore beaucoup plus de labilité sémantique dans les métadiscours des sujets profanes, simples apprenants, ou natifs désirant aider des étrangers. Quelle que soit leur adéquation aux réalités des faits de langue, ces métadiscours pédagogiques devraient être soumis, idéalement, à de constants ajustements de sens dans les interactions pédagogiques à l'Ecole, ou dans les séquences d'aides métalinguistiques en milieux dits "naturels" (Trévise, à paraître-b). Les métadiscours n'échappent pas aux contraintes des langues naturelles, puisqu'ils utilisent des langues naturelles.

Il semble ici plus opératoire de parler de métalexique, ou de métatermes, pour renvoyer au vocabulaire spécifique de description grammaticale, et de parler de métadiscours pour référer à la mise en œuvre de ces descriptions ayant clairement les langues et leurs fonctionnements pour objets, quel qu'en soit leur degré de sophistication. Ces métadiscours ainsi définis comme ayant pour seuls objets les codes linguistiques et leurs fonctionnements, leurs représentations aussi, souvent en termes de normes sociales, émanent de la conscience, et sont donc à différencier des multiples phénomènes plus complexes de réflexivité dans le langage.

\section{Spécificités de la connotation autonymique : le métalangage}

La langue de ces métadiscours reste très majoritairement une langue naturelle, même si, quand survient l'autonymie lexicale ou phrastique, quelques phénomènes sémantiques, morpho-syntaxiques, prosodiques ou typographiques lui sont spécifiques. On sait, à la suite de Rey-Debove en particulier, que l'autonymie provoque le blocage du sens, une impossibilité de synonymie et de traduction en langue étrangère. Elle citait 
l'exemple de la phrase quadruplement ambiguë Elle écrit toujours, qui produit en anglais She is always writing - She always writes - She is still writing, mais aussi She is writing toujours (1985 : 23). Elle ajoutait alors - en excluant, il est vrai, toute approche du code-switching - que si toujours n'était pas autonyme dans la dernière phrase, ce n'était plus une phrase anglaise acceptable, mais du babélien.

Ce que Rey-Debove appelle le métalangage a quasiment uniquement pour objectif la description d'un code linguistique donné, ce qui n'est qu'une partie de notre objet ici. Elle ne parle pas de métalangage ayant pour objet le fonctionnement de ce code en interaction, ou les représentations des sujets sur ce code ou un autre. Le métalangage tel qu'elle le définit, de façon donc restreinte, est pour elle un système sémantique qui présente des caractères formels, quand il signifie par iconicité. Elle souligne la grande ambiguïté des phrases qui peuvent concerner les mots et les choses, les mentions ou les usages. Les phrases métalinguistiques, ainsi définies, qui comportent de l'autonymie, utilisent donc des mots métalinguistiques (que j'appelle ici métatermes, comme adverbe, groupe nominal, etc.) qui n'ont pas de fonctionnements spécifiques, et des autonymes (toujours dans la dernière phrase plus haut). En français, tout autonyme est un groupe nominal masculin invariable :

"Le chien a aboyé" est bien construit en français.

"Parce que" est une conjonction.

Il n'y a là ni synonyme, ni traduction possible de l'autonyme et, si ces phrases ne sont pas repérées comme métalinguistiques, elles seront considérées comme syntaxiquement mal formées. A l'oral, la prosodie est parfois spécifique, et à l'écrit, des guillemets ou des italiques enlèvent le plus souvent toute ambiguïté.

Le discours rapporté en style direct est autonyme en ceci qu'il est censé être l'icône de ce qui a été produit, même si c'était incompréhensible.

Le métalangage, tel que le définit Rey-Debove, est donc un type de métadiscours particulier car il comporte des autonymes. Ce qui en fait linguistiquement la spécificité ne sont pas les métatermes, mais les termes autonymes. Seuls les autonymes engendrent en effet des phénomènes formels spécifiques, et tous les métadiscours ne sont pas du métalangage au sens restreint défini ici, car tous les métadiscours ne comportent pas de connotations autonymiques. En particulier, ceux qui ont pour objet les mises en fonctionnement des systèmes, les représentations sur les langues, sur les normes, le bilinguisme, l'apprentissage, utilisent véritablement une langue naturelle, non marquée. Le terme de métalangage me semble alors inapproprié, car décrivant des phénomènes restreints, et je le remplace par celui plus large, de métadiscours, dont une des manifestations est un métalangage spécifique utilisant l'autonymie. 


\section{Le métalinguistique}

On retrouve néanmoins bien sûr cette spécificité du métalangage, défini de façon restreinte, dans certains phénomènes de réflexivité, dans le métalinguistique donc:

- Qu'est ce que tu veux dire là par "perdu" ?

- Non, "perdu" n'est pas le bon mot. "Anxieux" je dirais plutôt.

Les autonymies, les mentions, sont très fréquentes dans les manifestations innombrables du métalinguistique, constitutives de l'activité langagière usuelle, et de la réflexivité propre au langage, de l'opacité dont parlait Culioli. Elles apparaissent génétiquement tôt, dès que l'enfant est capable de distinguer les mots des choses, et de dire que "train" n'est pas le mot le plus long qu'il connaisse.

Mais pour distinguer le métadiscours, homogène quant à son objet langue ou langage, du métalinguistique, reprenons l'exemple de Rey-Debove (1978 : 253) :

\section{C'est un marginal, comme on dit aujourd'hui.}

On voit que le métalinguistique est d'un ordre différent. Dans cette phrase, la préoccupation première du locuteur est d'identifier quelqu'un, et ensuite, par accroc pourrait-on dire, l'opacité du mot le fait réfléchir sur son adéquation, la mode, l'évolution du lexique, bref sur le mot lui même qui, d'usage, devient alors mention. La transparence disparaît, et l'opacification du dire donne cette double fonction au mot : usage et mention. Le signe lui-même devient objet de discours, par une boucle réflexive utilisant un verbe de dire. C'est ce que Culioli appelait "la prolifération du langage sur lui-même" (1973 : 87), tout en expliquant que :

(...) derrière l'apparent chaos d'un foisonnement sans contrôle, il se dégage une remarquable régularité dans les opérations par lesquelles des énonciateurs construisent un jeu structuré de relations et de références, produisant un surplus d'énoncés et modulant les significations. (1974:14)

L'exemple de Rey-Debove est donc de l'ordre du métalinguistique : il ne s'agit pas d'un discours dont l'unique objet est le code, ses mises en fonctionnement ou les représentations sur le langage. On en isole des bribes, comme Authier-Revuz (1996), Morel (1985), Gülich (1994) par exemple l'ont fait sur la langue des natifs adultes, ou comme d'autres l'ont fait dans les interactions entre natifs enfants et natifs adultes, ou entre enfants, ou entre natifs et non natifs, ou dans les productions de bilingues.

\section{Circonscrire le métalinguistique}


Selon les conceptions, le métalinguistique étant défini très largement comme tout phénomène de "distanciation" par rapport au dire, il peut aller jusqu'à englober la modalité (l'épistémique en particulier comme "mise à distance" de son dire, mais aussi l'appréciatif), les concessives, les reprises au subjonctif en français (le fait qu'il fasse beau...), tout énoncé repéré comme non assertif, éventuellement même négatif, puisqu'il faut dire une chose pour dire qu'elle n'est pas : on ne peut se contenter de ne rien dire. Il englobe plus traditionnellement le discours rapporté direct (tentative de reproduction des dires de l'autre) ou indirect (les dires de l'autre réassertés par le deuxième énonciateur).

Il s'agit là de possibilités qu'a le code de présenter une distance métalinguistique, de signifier l'intervention de l'énonciateur sur son propre dire, ou sur les dires d'autrui. L'énoncé de proverbes, ou autres extraits culturellement reconnaissables, peut également être considéré comme témoins d'une reprise de paroles. Toutes les imitations d'accent, d'élocutions, parodies et pastiches, mais aussi l'ironie, la complicité, rentrent aussi dans cette catégorie de distanciation intentionnelle.

Néanmoins, les limites deviennent floues quant à l'intentionnalité ou la conscience : une "contagion", involontaire, d'accent ne fait plus partie du domaine, et à la limite tout le dialogisme lexical, toute l'histoire des mots, véhicules et créateurs d'idéologie, rappellent toujours les discours précédents et à venir. L'énonciateur est inscrit dans une histoire et une idéologie et si je suis le premier auditeur du discours que je crée et planifie, les mots que j'emploie appartiennent à un passé dont les ramifications, la dynamique et la violence m'échappent (Lecercle, 1990). On sort du domaine du métalinguistique explicitement repérable dans le code.

Par contre, les différentes formes linguistiques des ajustements de sens, ces "mots qui ne vont pas de soi", liés aux négociations de sens avec soi-même (Authier-Revuz, 1995) et aux calculs de l'ambiguïté, liés aux représentations de la compréhension par l'allocutaire, l'organisation et la dynamique argumentatives (Borillo, 1985, Morel, 1985, Gülich, 1994), les reprises, et autres formes de rattrapages, les questions manifestant des incompréhensions ou des demandes de précision à l'allocutaire sont de l'ordre du métalinguistique explicite et observable.

Ces manifestations de la gestion du sens sont bien sûr beaucoup plus fréquentes à l'oral où la dynamique de l'élaboration des discours interactifs laisse ses traces ${ }^{2}$, ses marques.

\footnotetext{
2 "traces" est vraisemblablement une métaphore médiocre, puisqu'il n'y a pas une information première "salie" par des phénomènes de parole en gestation. Prendre en compte la dynamique d'élaboration même des pensées et des sens en interaction, c'est exclure une telle hiérarchisation et une telle chronologie induites par cette métaphore.
} 


\section{Les degrés de conscience}

Le problème qui se pose néanmoins pour l'emploi du préfixe méta- est le degré de conscience que l'on suppose dans ces usages si variés. Dans le cas de la modalité, par exemple, il s'agit le plus souvent sans doute d'un travail épilinguistique, défini comme l'activité métalinguistique non consciente. Il est bien délicat de statuer sur le degré de conscience attaché à toutes ces manifestations de la réflexivité inhérente au langage et à sa pratique.

Il est tout aussi complexe de relier l'intuition des natifs à un degré de conscience défini, même si l'on sait qu'il est bas, et que les natifs ne sauraient expliquer le plus souvent pourquoi ils acceptent ou rejettent tel ou tel énoncé, "sentiment" qui ne correspond d'ailleurs pas forcément avec leurs propres pratiques. Les représentations des normes linguistiques et sociales se confondent fréquemment et sont souvent non conscientes.

\section{Spécificités formelles du métalinguistique}

En dehors des phénomènes d'autonymie que nous évoquions précédemment, il existe des régularités observables dans l'emploi du métalinguistique en interaction, dans ces manifestations de la réflexivité opacifiante. Morel a étudié, dans un corpus oral, des régularités syntaxiques intéressantes, qu'elle qualifie de typiques du métadiscursif (en particulier les syntagmes infinitivaux introduits par pour, comme dans pour employer un mot à la mode, ou l'emploi de certaines conjonctions, $1985: 108,114-115)$ et elle a aussi isolé la structure de prolepse qui est bien du domaine métadiscursif, comme dans l'exemple qu'elle donne :

j'ai vu un Américain aujourd'hui qui me disait, tu me diras c'était un Américain oui mais il m'disait que... (Ibid. : 113)

Cette construction syntaxique est particulière en ce qu'elle comporte deux propositions coordonnées par mais (ou mais enfin), et présente un marqueur spécifique dans la première proposition qui interrompt le développement de l'énoncé initial.

L'ouvrage d'Authier-Revuz étudie en détail ces manifestations de l'hétérogénéité discursive du métalinguistique. Il n'y a pas une langue-objet, mais un tissage de l'hétérogène, du dire d'une pensée et des proliférations sur ce dire qui participent aussi à l'élaboration de la pensée.

Les tentatives de clarifications terminologiques se heurtent vite à l'artificialité des limites à donner à ce que recouvrent les notions, et qu'elles dépendent des décisions, peut-être arbitraires, de l'observateur. 


\subsection{Une conception du langage}

L'ensemble de ces manifestations spontanées au sujet de son propre dire, ou du dire de l'autre, cette "auto-représentation du dire en train de se faire" (Authier-Revuz, 1995 : 18), entraîne à considérer que le langage est certes un système de représentation du monde, mais aussi une représentation de lui-même et une expression personnelle du sujet qui actualise sa "propre" pensée et sa propre représentation du monde à travers un code commun dont il sent souvent l'opacité voire l'inadéquation, avec une conscience plus ou moins grande du dialogisme. Le langage, dans son essence, a cette réflexivité, cette faculté de glose sur lui-même qui s'actualise plus ou moins suivant les sujets, ou les types d'interactions dans lesquelles ils sont impliqués.

Authier-Revuz écrit à propos de son travail :

L'objet visé - cette configuration énonciative, tant au plan formel que comme élément de l'activité langagière - relève de plusieurs champs vastes et travaillés par des problématiques diverses et parfois opposées ; celui - en tant que forme d'auto-représentation du dire - du métalinguistique, pris ici au sens le plus général d'engageant "de la" représentation d'objets de nature langagière ; celui - en tant que mode dédoublé, distancié,... non-un du dire - de "l'énonciation" en général, évidemment, mais plus particulièrement de ce qu'on pourrait appeler la "complexité énonciative", c'est-à-dire l'énonciation conçue, observée, comme n'étant pas "une", "mono-bloc", et dont l'approche comme plurielle ou (et) hétérogène ne peut pas ne pas engager des théorisations du sujet, du sens et de la communication. (Ibid. : 1)

Et il n'existe pas de position hiérarchiquement plus élevée que le langage pour en parler. C'est un des sens de l'aphorisme lacanien "il n'y a pas de métalangage" : le fait que nous soyons obligés d'utiliser le langage pour parler du langage, "est justement ce qui prouve que nous ne quittons pas celui-ci" (Lacan, cité par Authier-Revuz, Ibid. : 13).

C'est donc bien d'une conception du langage vu comme autre chose qu'un simple instrument de communication d'un contenu informatif qu'il est question ici. Il s'agit d'une théorie du "sujet marqué d'un clivage interne à lui-même, par le fait du langage dont il est le produit" (Ibid. : 48).

Citons encore Authier-Revuz :

(...) dans le discours qui est tenu par les énonciateurs au sujet de ces "fragments" de la chaîne qu'ils énoncent, on verra que ce réel suffisamment "résistant" au dire pour en suspendre localement le mode standard du "qui va de soi", ouvrant dans le dire des points de non-coïncidence que les commentaires, qui en donnent une représentation, reconnaissent et "gèrent" tout à la fois, ne saurait être ramené (...) aux seuls faits de l'interlocution avec cet autre à qui l'on parle ou de l'interdiscursivité (...) : crucialement, au-delà des paramètres "dialogiques" de non- 
coïncidence représentés par les gloses, celles-ci disent que ce à quoi l'énonciateur a affaire dans son énonciation c'est au fait de la langue - de la langue éprouvée, rencontrée doublement, dans sa raideur, de système de différences, de structure ouvrant un manque au cœur de la nomination, et dans sa profusion instable d'espace d'équivoque ouvrant sur un excès de sens, et au double réel de laquelle se dit dans les gloses le rapport du sujet, et le mode, essentiel lorsque c'est d'être parlant qu'on est sujet, sur lequel il s'y inscrit et il s'y joue. (Ibid. : 58)

Benveniste l'écrivait : "Bien avant de servir à communiquer, le langage sert à vivre" (1974 : 217). Les boucles réflexives du dire ne sont pas seulement des manifestations de contrôle mises en œuvre dans la communication, où un sujet transmettrait l'habillage pur et simple d'une pensée préexistante. Le sujet est en même temps un être de langage et en même temps il subit la "violence du langage" (Lecercle, 1990), et surtout peut-être dans "cet écart interne que creuse dans l'énonciation l'autoréception qui la traverse (Authier-Revuz, Ibid. : 153).

Cette notion large, multiple, du métalinguistique interdit ce que permettait celle de métadiscours plus constitué, mais tout aussi large, ou gradué, car ayant une multitude de formes, de degrés de scientificité et d'objets : il interdit de se représenter un niveau 1 de la langue qui parlerait directement des choses, et un niveau 2, implicitement déclaré supérieur, ou postérieur, d'où on pourrait parler de ce niveau 1, qui serait du langage sur ce langage primaire.

C'est pourquoi le préfixe méta-, apparu chez Aristote dans le mot métaphysique, renvoyant à la science qui "vient après" la physique, est vraisemblablement impropre pour parler du métalinguistique. Il reste sans doute approprié, par contre, pour les métadiscours, qui ont plus clairement un objet extérieur.

Mais, on retrouvera du métalinguistique, bien évidemment, dans les métadiscours (surtout oraux) qui sont eux-mêmes des manifestations langagières, et qui n'échappent donc pas à la réflexivité, aux ajustements de sens et aux modalisations et marques d'argumentation diverses. On les retrouvera aussi dans les méta-métadiscours. Les boucles s'accumulent...

Dans ce volume, F. François préfère, à cette conception verticale d'une hiérarchie de discours emboîtés, une conception dialogale du métalangage comme commentaire et, en particulier, comme commentaire intégré, inhérent à la signification du discours. L'auteur rappelle qu'avant de se trouver lié de façon frontale à son objet, tout discours se trouve lié de multiples façons à d'autres discours plus ou moins lointains, et à des discours futurs, anticipés ou non, qui modifieront la signification des discours passés. Il parle, à propos de l'acquisition du langage, d'un "dialogue réflexif intégré", un 
métadiscours implicite (François, 1994), partie prenante de l'usage même des signes (conduites d'imitation, "c'est pour rire", "je fais semblant", clins d'œil aux adultes, paraphrases et reformulations quand l'adulte ne comprend pas), et insiste sur le rôle du corps de l'enfant comme porteur de commentaire.

François citait par ailleurs ces réponses possibles à la question $Q u ' e s t-c e$ que tu veux manger?

- du pain

- de la langouste au chocolat

- c'est pas l'heure

- toi mon amour

- je bois tes paroles

- tu me tutoies maintenant?

- vouloir vouloir t'en as de bonnes. Il ne suffit pas de vouloir.

Et il posait la question : laquelle ou lesquelles de ces réponses est métalinguistique?

Et c'est encore F. François qui distingue entre ceux qui savent faire la cuisine, en jouir et en parler. Tout sujet parlant sait faire les trois à propos du langage. Il en souffre aussi.

Danon-Boileau (1994 : 71) insiste également sur "le méta comme faculté de détour". Les signes ouvrent la possibilité de faire des opérations, des détours qu'on ne peut faire avec les objets. Il distingue lui aussi deux types de méta : les mécanismes (les procédures) méta d'une part et les représentations (les théories) méta de l'autre (Ibid. : 71). Il défend comme nous l'idée que ce qu'il appelle la théorie "méta" d'un savoir-faire "n'est pas le second temps réflexif, la glose a posteriori sur ce savoir faire" (Ibid. : 72), et donne l'exemple d'un détour méta grâce auquel un sujet peut remédier aux ratés d'un mécanisme défectueux. Il s'agit d'un jeune homme qui pouvait expliquer le sens figuré du mot girouette ("quelqu'un d'inconsistant") sans pour autant, seul, en définir le sens propre, car il présentait des troubles de l'organisation visuo-spatiale. Le sens figuré, ne mettant pas en jeu la représentation de l'espace, lui a permis, avec l'aide du thérapeute, de se servir de cette fonction du méta pour situer ce qu'il savait faire par rapport à ce qu'il ne savait pas faire, en fournissant une représentation cohérente de ce que peuvent être les différents sens du mot. "La réflexion sur un mécanisme peut donc précéder la mise en jeu convenable du mécanisme auquel elle s'applique" (Ibid. : 74). Et DanonBoileau de conclure :

(...) la réflexion "méta" n'est pas nécessairement une glose pédagogique sur un mécanisme qui fonctionne. Au contraire, la mise en circulation d'un enjeu méta 
dans les échanges avec l'enfant permet l'affermissement de mécanismes encore fragiles. Il permet surtout à l'enfant de tolérer les "ratés" de sa communication en leur construisant un sens. Ils trouvent ainsi à s'intégrer dans l'histoire et la théorie de l'échange. Au prix d'un détour nécessaire, le méta devient alors une façon d'articuler les discontinuités sans les annuler. (Ibid. : 77)

Ces considérations sur le langage, sa nature, sa complexité, et ses modes de mise en œuvre, s'attachaient dans un premier temps à clarifier le champ du "méta", qu'il recouvre les métadiscours pleinement conscients, ou les multiples formes du métalinguistique plus diffus, et omniprésent à l'oral, et, vraisemblablement, dans les discours intérieurs. Après une brève incursion du côté de la jouissance, j'en viendrai aux phénomènes non observables liés à l'acquisition.

\section{Jouissance métalinguistique}

L'expression est de Culioli (1990 : 41), mais je la reprends volontiers à mon compte. Il référait au sujet énonciateur qui s'adonnait, par les jeux de langage de tous ordres, à cette jouissance du métalinguistique.

Dans une société où les Guignols de l'Info ou les Zap'tualités font un audimat extraordinaire, la jouissance métalinguistique est désormais une valeur médiatique sûre. Les imitateurs jouent en effet notamment sur la parodie, les timbres de voix, les accents, l'ironie, les tics, les tons, les différentes façons de parler des footballeurs ou autres patineurs et chanteurs, en même temps que sur la langue de bois des politiciens, et donc parodient aussi les contenus pseudo-informatifs ou au contraire forcent le trait d'un discours partisan, se jouant ainsi des jeux et enjeux de paroles publiques. Ils changent dès lors les regards et les écoutes que l'on porte sur les originaux eux-mêmes, écoutes qui deviennent plus métalinguistiques. C'est une véritable éducation métalinguistique des téléspectateurs qui prennent conscience qu'ils avaient effectivement, plus ou moins consciemment, remarqué ces phénomènes.

On retrouve cette jouissance chez les enfants, sous diverses formes, et même parfois à l'Ecole, dans les trop rares occasions où l'on tente de les faire vraiment réfléchir sur le langage ou la langue maternelle ou étrangère et donc retrouver des activités ludiques.

Et que penser de ces formules à l'allure de dénégation : je te dis pas, je te raconte pas qui précèdent si souvent les récits des jeunes Français ? Sont-elles dues à des verbalisations de sentiments de l'indicible, ou de l'inadéquation du dire en tout cas ? La jouissance se transforme vite en douleur quand on a le sentiment que les mots "manquent", ou qu'on n'arrive pas à exprimer ce qu'on ressent, quand "les mots ont 
dépassé la pensée", quand les enfants n'arrivent pas à trouver le bon mot, quand les malentendus sont révélés (Trévise et Deprez-de Hérédia, 1984).

C'est que le langage n'est pas juste objet pour linguiste, il est l'identité même des sujets inscrits dans une histoire et une idéologie. Et c'est ce qui va différencier l'acquisition du langage de celle des langues 2, dénommées ainsi car elles ne sont que deuxièmes, et n'auront, dans la majorité des cas, pas participé à l'élaboration des personnalités, des représentations du monde et du langage (Trévise, 1994). Toutes les manifestations de la réflexivité, de même que la connaissance du monde, ne seront pas à réacquérir dans leurs fonctionnements. "Seules" devront être acquises les formes correspondantes dans l'autre langue, elle-même pétrie d'une autre culture.

L'incursion dans les pathologies, avec Jakobson, ou Danon-Boileau, nous amène aux descriptions de ces mêmes phénomènes dans d'autres situations de communication, qui entraînent un recours spécifique, et souvent fonctionnel, au métadiscours et au métalinguistique, situations qu'avait d'ailleurs mentionnées Jakobson : les situations d'acquisition du langage (et donc de cette réflexivité), et les diverses situations de rencontre d'une langue 2 par la simple audition d'une langue inconnue ou par l'acquisition d'une langue 2. S'ajoutent à cela les phénomènes métalinguistiques liés aux diverses formes de bilinguisme, dont le code-switching, et autres formes transcodiques, qui ne sont malheureusement pas envisagées dans ce volume (cf. Deprez, 1994, et Schlyter, 1995).

\section{Réflexion, réflexivité et acquisitions}

Etudier les pathologies et les phénomènes liés à l'acquisition, Jakobson l'avait compris, illustre négativement bien des choses sur le fonctionnement langagier/métalangagier entre adultes natifs. C'est pourquoi il faut définir les spécificités observables liées à l'acquisition du langage, au développement et à la pratique du bilinguisme, à l'acquisition et la pratique des langues dites "étrangères", à l'Ecole ou hors Ecole.

Cette étude des spécificités évitera ainsi de définir, pour forcer le trait, les bilingues ou les apprenants de L2 comme manifestant une conscience métalinguistique : c'est le cas de tout locuteur natif, enfant ou adulte. L'intensité dépendra vraisemblablement plus des sujets que des situations dans bien des cas, et ne saurait à elle seule, en tout cas, isoler une spécificité. Par contre les modes d'expression de ces activités métalinguistiques, qui exploitent des connaissances métalinguistiques, auront des formes particulières dans les différents cas d'apprentissage ou de bilinguisme. 
Il est d'ailleurs une activité métalinguistique qui sera bien difficile à acquérir en langue étrangère, même en activité de compréhension, ce sont les jeux de mots. Les apprenants adultes ne rencontreront pas les mêmes difficultés que les enfants en langue maternelle qui ont parfois insuffisamment de recul métalinguistique pour comprendre. C'est le jeu même qui leur échappera, son intégration à la culture cible, mais aussi la dissociation des signifiants et des signifiés et la maîtrise des ambiguïtés phoniques par exemple, surtout si l'apprentissage est passé par l'écrit. La polyphonie change de nature. Et la traduction exacte des jeux sur les mots est impossible, comme celle de l'autonymie.

La question des impacts des activités métalinguistiques dans les processus d'acquisition est encore un débat ouvert, dans lequel j'adopte la position de la croyance en l'utilité de représentations (ou connaissances) métalinguistiques "correctes", c'est-àdire adéquates aux réels linguistiques et formulées dans des discours métalinguistiques interactifs, ajustables, négociés, en un mot réellement compréhensibles et susceptibles d'être appropriés, voire utilisés par les sujets auxquels ils s'adressent (Trévise, à paraitreb).

Je renvoie, pour la question de la nature et de l'efficacité de l'aide plus interactive, moins programmée, du natif en L2, à l'article de M.-T. Vasseur et J. Arditty dans ce volume.

\subsection{Mouvements cognitifs : activités métalinguistiques et épilinguistiques}

Les observations faites dans les divers types d'acquisition/apprentissage montrent que les apprenants ont une activité métalinguistique, quel que soit le degré de sophistication atteint par leurs représentations métalinguistiques, et quelle que soit l'adéquation de cette activité et de ces représentations à la réalité linguistique, à la fois de leurs propres systèmes de production/compréhension et des systèmes source et cible.

"Représentation" réfère à une notion hétérogène : les sujets construisent des représentations sur les codes linguistiques, leurs fonctionnements, leurs compétences personnelles, les objectifs de l'apprentissage, mais aussi donc sur les normes, les difficultés des langues, de l'apprentissage, sur les "bonnes" stratégies d'apprentissage, sur le rôle de la langue maternelle, sur les types de tâches et d'interactions dans lesquelles ils sont impliqués dans la dynamique de l'apprentissage. Ces représentations sont à la fois variées, fluctuantes, et plus ou moins justifiées. Certaines échappent à une maîtrise consciente claire, en particulier celles qui touchent aux images des langues et des pouvoirs en jeu. On ne peut en faire une liste qui soit exhaustive, ni a fortiori rendre compte de leurs rôles. Le non-conscient affectif vient de plus se mêler au nonconscient cognitif. 
L'étude des malentendus, mais aussi les observations de séquences de "contrats pédagogiques" montrent que l'activité métalinguistique, en particulier d'attention aux codes, est omniprésente, parfois de façon diffuse, mais qu'il serait illusoire de croire qu'elle est efficace dans tous les cas. On peut néanmoins penser que lorsque l'attention et donc la conscience des apprenants - est attirée, pour une raison ou pour une autre, sur la langue, et plus précisément sur les causes formelles d'un dysfonctionnement dans la communication, cette prise de conscience devient un moteur de l'acquisition, ou du moins une amorce motivée de rééquilibrage du système interlinguistique, si toutefois les sujets ressentent ces dysfonctionnements comme gênants. Les apprenants ont alors leur attention attirée, par ce type de feedback, sur les différences entre leurs productions ou leur compréhension et les formes cible, et il peut s'ensuivre une modification de leurs hypothèses conscientes ou non conscientes (cf. la notion de "règle critique" proposée par Klein, 1986).

On se reportera à l'article de D. Huot et R. Schmidt, dans ce volume, qui distinguent entre les différentes formes de conscience suivant que la notion s'applique à la pensée ou aux contenus de pensée : prise de conscience, conscience comme intention et conscience comme connaissance.

\section{Contrôle en production}

L'activité métalinguistique est, sans nul doute, plus importante quand on aborde un deuxième système linguistique, mais personne ne cerne sa ou ses natures exactes, ni surtout son rôle dans l'acquisition. Il est difficile de définir ce qu'on appelle le "contrôle" en production (ou en compréhension d'ailleurs, bien que le domaine de la compréhension soit encore trop ignoré dans la littérature, cf. néanmoins Bremer et al., 1996), autrement que comme un type d'activité complexe, qui se produit en quelques fractions de secondes.

Certains auteurs ont tenté de mesurer les pauses, les "blancs" dans la production (Ellis et Rathbone, 1990), pour tenter de mesurer ce qui constituerait les marques d'un contrôle. Mais peut-on vraiment penser que la longueur des pauses est un paramètre qui indiquerait un contrôle quand on n'a pas de réelle théorie de la production qui assignerait des unités de temps à des processus mentaux ? Quel est le temps requis pour se référer à une règle grammaticale particulière, pour trouver un mot dans son lexique, pour planifier une argumentation ? Le temps nécessaire varie-t-il suivant les types de règles, le contexte, la forme de représentation de ces règles (sous forme d'exemple, d'explication, de traduction plus ou moins consciente, cf. Robinson, 1996) ? Le monitoring, pour reprendre le terme de Laver (1973), est typiquement une activité à laquelle le chercheur ne peut avoir accès. C'est certainement une activité complexe et 
subtile, qui obéit à différentes sortes de contraintes mémorielles, psychologiques, sociales et institutionnelles, que même l'auto-observation à chaud n'éclaire que très partiellement.

\section{Transferts métalinguistiques}

En L2, ces mouvements incorporent de façon complexe les deux systèmes linguistiques en présence, ce qui va donner lieu à des transferts analogiques - positifs ou négatifs, - d'activités et/ou de représentations métalinguistiques. Ces transferts vont donner en particulier naissance à des relations bi-univoques entre les deux systèmes, relations qui ne seront pas toujours adéquates aux réels linguistiques, mais qui viendront alimenter les représentations métalinguistiques des sujets sur la langue 2. Les apprenants auront donc non seulement des conduites linguistiques contrastives, ou assimilatrices, suivant leurs sentiments de distance entre les langues (Kellermann, 1978), mais ils auront aussi nécessairement des représentations liées sur les deux langues, tremplins ou handicaps suivant les cas.

Des représentations (parfois fausses) sur la langue maternelle sont ainsi transférées dans des relations bi-univoques français-anglais, par exemple, imparfait/prétérit en $\mathrm{BE}+$ -ING, et donc dans le système de représentations sur la langue 2. Le fait qu'il y ait ainsi transfert n'est pas toujours conscient et verbalisable manifestement, même si certains transferts sont de fait encouragés par un enseignement trop hâtivement contrastif, et qui d'ailleurs, généralement, ne se conçoit lui-même pas comme tel (Trévise, 1994, et à paraître-a).

\section{Automatisation : de la conscience à la non conscience}

Un des objectifs de l'acquisition est l'automatisation des règles en production et en reconnaissance, quand connaissance de règles il y a. Ce mouvement concerne le passage de la conscience à la non-conscience, et peut seul assurer une maîtrise réelle de la production et de la compréhension sans surcharge cognitive, du moins quand les règles du produit sont bonnes. (On se reportera à l'article de J.-E. Gombert, dans ce volume, qui propose une séquence de développement métalinguistique en quatre phases, dont la dernière est l'automatisation des comportements métalinguistiques.)

Cette automatisation entraîne un mouvement des règles conscientes au départ vers le domaine de la non-conscience, où elles rejoignent donc l'ensemble hétérogène des règles de production qui ne sont jamais passées par la conscience : tout ce qui relève de l'automatique, ce qui est géré par l'activité épilinguistique, tout ce qui n'a pas été analysé par l'apprenant, tout ce que l'enseignement n'a pu lui enseigner, car tous les phénomènes syntactico-sémantiques ne sont pas percés à jour dans leurs fonctionnements discursifs et interactifs par les linguistes (cf. l'article de S. Haller et B. Schneuwly dans ce volume, qui conclut à la rareté des régulations métalinguistiques 
observables dans une tâche de rédaction d'un texte argumentatif). On peut enseigner la morphologie plus facilement que la totalité des tendances à l'œuvre dans la représentation aspecto-temporelle d'une langue par exemple. Toutes les "règles" ne sont pas égales entre elles, ni en complexité, ni en fréquence relative dans telle ou telle langue.

L'enseignement joue un rôle certain dans l'élaboration des représentations des apprenants. En effet, l'origine de leurs représentations conscientes peut apparaître par une analyse de la métalangue et les notions qui sont très souvent véhiculées par les manuels et/ou le discours enseignant.

Mais que l'enseignement ait un impact sur les représentations métalinguistiques n'implique pas nécessairement que, par ce biais, il ait réellement un rôle efficace dans les processus d'acquisition, et encore moins que cet impact éventuel soit le même pour tous les apprenants, à n'importe quel stade acquisitionnel de structuration du nouveau système.

La littérature qui rend compte d'expériences de comparaisons sur des ordres d'acquisition en milieu naturel et à l'Ecole est peu convaincante, et les défauts méthodologiques des expérimentations sont assez flagrants (pour un compte-rendu, voir par exemple Ellis, 1990, Widdowson, 1990). En particulier, ces études ne mentionnent pas le type d'enseignement reçu à l'Ecole, le type d'analyse linguistique sur lequel ces explications et leur métalangue s'appuient. On constate, après un "enseignement" (qu'on ne décrit pas), si telle structure ou tel ordre des mots est appris, c'est-à-dire est produit (et jamais "compris") dans $90 \%$ des contextes obligatoires (et dans des types de tâches très métalinguistiques de nature).

Or on sait qu'"enseigner" tel ou tel point de "grammaire" peut vouloir dire maintes choses extrêmement différentes, et qu'il se pourrait que la variation dans le type d'enseignement entraîne une variabilité dans les productions. On sait aussi que plus d'une explication métalinguistique est "mauvaise", en ce sens qu'elle ne rend pas compte de la réalité, non seulement du code, mais du fonctionnement de la langue cible d'une part, et que, d'autre part, elle ne tient pas compte de l'"état des lieux" des représentations au moment de cet enseignement.

Sur la différence entre ce qu'il nomme "acquisition spontanée" et "apprentissage par enseignement", Bresson écrivait :

Ces deux modalités sont bien connues des informaticiens de l'Intelligence Artificielle : l'opposition entre procédural et déclaratif. On a étudié ces dernières années, les conditions cognitives de ces deux façons d'apprendre et les résultats qu'on en peut obtenir. On a montré ainsi les différences entre les règles procédurales que ce savoir applique et celles que l'on peut apprendre par 
enseignement verbal, les différences entre les informations dont on a une conscience qui permet de les communiquer, et les savoirs qui, eux, demeurent implicites et dont le système de règles peut être différent.

Les difficultés de l'enseignement des langues étrangères tiennent largement à ces problèmes. Les règles que l'on doit acquérir pour produire la parole doivent fonctionner de manière procédurale : on doit parler, entendre, comprendre sans avoir à formuler ou à choisir ces règles, mais produire leurs résultats. Cependant la transmission, au moyen de la communication verbale, des savoirs qui correspondent à ces règles, pose le problème de composer leur acquisition procédurale avec l'enseignement déclaratif de certaines d'entre elles ${ }^{3}$. (1991: 23)

Tout le problème est de savoir si les règles enseignées par enseignement déclaratif (métalinguistique), et les connaissances déclaratives des apprenants, "correspondent" aux règles du fonctionnement linguistique. Si ce n'est pas le cas, il serait alors souhaitable que la "composition" dont parle Bresson ne se fasse pas. On ne peut enseigner des règles de production, et il semble clair qu'il vaut mieux ne pas enseigner de règles que d'enseigner des règles du produit fausses, ou trop parcellaires, ou encore mal formulées par rapport aux représentations des apprenants qui tentent de les comprendre et de les assimiler.

La vraie question demeure néanmoins : un "bon" déclaratif, c'est-à-dire un "bon" métadiscours sur la réalité du fonctionnement de la langue 2, ou des deux langues 1 et 2, "bien" simplifié et adapté aux représentations métalinguistiques préalables des apprenants, peut-il jouer un rôle dans les processus d'acquisition/apprentissage ? Ce rôle pourrait alors soit être régulateur en termes de mémoire et de contrôle possible (dont la nature reste obscure), post- ou pré-opératoire, soit permettre l'automatisation. Mais on sait que les "bons" métadiscours restent encore largement à élaborer, même si de rares manuels d'anglais $\mathrm{L} 2$, ou de français langue maternelle commencent à témoigner des résultats de telles préoccupations didactiques.

Une fois défini, et contrôlé dans ses effets, ce que l'on entend par "enseignement métalinguistique", il reste que, en dehors de la formation intellectuelle des apprenants, un argument majeur en faveur de cet enseignement, ou du moins d'une réflexion métalinguistique guidée, est le fait que bien des migrants ne dépassent en général pas un stade dit "initial", et que les programmes d'immersion ont montré que si la fluency des apprenants était bonne, l'accuracy ne l'était pas toujours, loin s'en faut, et qu'il n'y avait donc pas de système de pré-correction ni de post-correction d'"erreurs".

\section{Composition de l'automatisation et de l'automaticité}

Une grande partie des phénomènes d'apprentissage, en L2 comme en L1, échappe à la conscience : non seulement les phénomènes liés à la pragmatique, la cohérence

\footnotetext{
3 souligné par moi.
} 
sémantique et la connaissance du monde, la variabilité des interprétations suivant les contextes, mais aussi les formes mêmes de réflexivité, et bien sûr bon nombre de règles formelles et de leurs domaines d'application, dont la linguistique, à l'heure actuelle, ne sait pas rendre compte.

Il est donc fort heureux qu'il y ait apprentissage sans conscience, de façon non analysée, avec parfois, selon les cheminements, des accès à une analyse consciente postérieure des règles utilisées, mémorisées et généralisées par analogie. Ainsi les considérations sur la conscience ne doivent pas faire occulter le fait que bien des choses en acquisition sont de l'ordre du non-conscient.

Les mouvements cognitifs vont donc dans les deux sens, et ne drainent vraisemblablement pas les mêmes règles : d'une part de la conscience à l'automatisation, avec possibles retours et recours à des règles conscientes mémorisées, en cas d'obstacles par exemple, et d'autre part de l'automatique, du non-analysé à une possible conscientisation partielle et progressive, spontanée ou guidée. Les deux cheminements ne sont vraisemblablement pas du même ordre : la conscientisation ne modifie guère les savoir-faire automatiques préalablement installés. Elle permet cependant sans doute la progression à long terme vers un stade plus avancé. Et on ne sait pas si l'automatisation de règles conscientes les fait entrer dans le travail épilinguistique global de structuration du système intermédiaire et de test des hypothèses non conscientes.

On cerne donc mal à ce jour comment on peut passer de savoirs métalinguistiques (quelle que soit leur adéquation) aux savoir-faire (quels qu'ils soient) largement automatisés, et au contrôle des effets de sens, à ce qu'on appelle souvent l'appropriation. On cerne tout aussi mal comment se gère l'intégration des savoirs automatisés et des savoir-faire automatiques, sur lesquels peut s'exercer une réflexion ultérieure, bref la "composition" dont parlait Bresson plus haut. Cette composition est l'œuvre d'une activité épilinguistique qui gère les rencontres de tous ces cheminements. Elle échappe à la conscience. L'articulation complexe entre des processus d'apprentissage conscients et non conscients échappe à la conscience, et elle est méthodologiquement bien difficile à approcher.

Les savoir-faire traduisent la nature de règles de production et de compréhension. La connaissance métalinguistique se nourrit, elle, de règles des produits issues, $a$ posteriori, de l'observation des produits soit en milieu naturel, soit à l'Ecole, ou issues de l'enseignement de règles régissant les produits à l'Ecole. La "composition" concerne donc l'intégration, ou l'articulation, des deux types de règles. Les descriptions grammaticales ne sont pas des descriptions de l'activité langagière (cf. Besse et Porquier, 1991), ni des règles d'effectuation de la production, ni, encore moins de la 
compréhension, on le sait, même si elles tentent de les "simuler", pour reprendre les termes de Culioli. On ne sait pas encore décrire ni expliquer les va-et-vient cognitifs des représentations métalinguistiques vers les activités méta- et épilinguistiques, ni la "composition" des deux domaines dans la dynamique de la mémorisation à court et/ou à long terme.

Il reste que l'activité métalinguistique exercée par tel ou tel sujet peut n'avoir qu'une influence très négligeable sur sa progression, comme le même type d'activité métalinguistique, ou un autre, peut avoir une influence pour un autre sujet (qui aurait un autre profil cognitif, ou une autre motivation), et se "composer" avec ses hypothèses non conscientes, ou ses activités de mémorisation de bribes non analysées. Il est bien délicat de généraliser, et on est encore loin de la découverte d'invariants d'acquisition précis dans ce domaine (cf. M.-T. Vasseur et J. Arditty dans ce volume).

\subsection{Les métadiscours implicites à l'Ecole}

L'Ecole est le lieu privilégié pour les activités de type métalinguistique. Mais certaines se dissimulent, engendrées par l'inévitable simulation d'interactions "authentiques". D'autres sont réputées ne pas exister sous prétexte qu'on n'en voit aucun indice.

Même quand il n'y a pas de métadiscours explicites, d'explications grammaticales sur le code et le fonctionnement linguistique en discours, c'est très fréquemment la langue qui est l'objet implicite des cours de langue maternelle ou de langue étrangère. Tout enfant adapté socialement à l'Ecole comprend vite que les questions du maître ne sont pas des vraies questions, qu'une rédaction ne doit pas raconter effectivement comment on a vécu l'arrivée de l'automne, mais doit se conformer aux normes du "français des dimanches". Il aura donc compris que la langue est objet, et que si le cours d'anglais a porté sur must, il devra employer le maximum de must, quitte à raconter n'importe quoi. Il devra s'identifier à John ou Mary, et employer les déictiques en conséquence, oublier qu'il a une identité forgée par le français, et se plier au formalisme de ces jeux de langues contraints. C'est cet ensemble de phénomènes que j'avais regroupé sous l'étiquette de "simili-énonciation", en en soulignant l'artificialité obligée, et le fonctionnement normatif (Trévise, 1979). Même la langue maternelle se dilue dans ces cadres de jeux formels, et les enfants sont capables de produire des monstres syntaxiques ou sémantiques dans leur propre langue, car elle ne signifie plus alors pour eux, et leur intuition de natif ne s'exerce plus.

On peut faire entrer ces phénomènes dans les métadiscours cachés, implicites, sortes d'attitudes métalinguistiques contraintes, de distanciation nécessaire par rapport aux dires, ou aux écrits. Le français langue maternelle à l'Ecole en vient à avoir des 
points de similitude avec une langue étrangère, et on ne peut manquer d'être surpris d'entendre tous ces jeunes francophones répéter qu'ils sont "nuls" en français.

Un autre type de métadiscours implicite est aussi spécifique de l'Ecole : c'est ce que recouvre le mythe du non-discours métalinguistique des partisans de l'approche dite communicative stricte. Même si l'on ne prononce pas un seul mot d'explication métalinguistique en classe, même si l'on ne présente que des situations réputées "authentiques" de communication, on intervient néanmoins dans le processus d'apprentissage de par la sélection de ces types d'"input" et de situations, et la progression plus ou moins ordonnée que l'on choisit.

En outre, à l'Ecole comme ailleurs, en L2 comme en L1, on ne peut, de toute évidence, empêcher que les apprenants aient un métadiscours intérieur et une activité métalinguistique inhérente à toute activité d'appropriation d'un système linguistique. Cette activité métalinguistique a lieu, même si elle n'est pas, ou ne peut être, verbalisée. Ce n'est pas parce qu'il y a silence qu'il n'y a qu'activation des activités épilinguistiques, ou, d'ailleurs, qu'il y a de telles activités, comme, a contrario, ce n'est pas parce qu'il y a silence, ou pause, qu'il y a contrôle métalinguistique conscient, utilisation des connaissances métalinguistiques verbalisables (une fois que l'apprenant s'est forgé une représentation du besoin de contrôle).

\subsection{Fossilisations métalinguistiques et métalangue de bois}

J'ai décrit ailleurs (Trévise, 1993, et à paraître-a et b) les fossilisations métalinguistiques issues des métadiscours de l'enseignement que révèlent maintes verbalisations des apprenants francophones, en classes de français langue maternelle ou de langue étrangère. Leur "passé métalinguistique" intense, à propos de la L1 comme de la L2, leur fait produire, même devant des faits de langue de français, des métadiscours plaqués, régurgités, qui fonctionnent à vide, sans souci de référenciation. A ces métadiscours non adéquats aux réels linguistiques, et qui ne fonctionnent pas, à aucun sens du terme, on peut opposer les véritables discours issus de raisonnements, qui ne sont plus de simples résonances, et qui eux pourraient avoir un rôle dans l'acquisition.

Les métadiscours souvent récités par les apprenants ne gardent des fonctions du langage ni la fonction de représentation symbolique créatrice de pensée, ni l'ajustement à l'objet de discours et à l'autre. Ils instancient une vacuité de pensée, et ne "représentent" manifestement rien pour eux. L'incohérence n'est plus un obstacle qui ferait surgir des phénomènes de conscience. Cette non-relation entre des mots simplement enfilés comme des perles et une signification fait penser, sans la rouerie, mais avec le même désir de combler ce qu'ils pensent être des attentes, à la langue de 
bois de certains politiciens. On est loin des vertus pédagogiques que Lewis Carroll attribuait au non-sens : vertus de réflexion sur le langage, source, et non simplement medium et objet, d'enseignement.

Le discours métalinguistique de l'Ecole, quand il représente une métalangue parcellaire, et inadéquate, mais rabâchée et mémorisée, a ceci de particulier qu'il est normatif (le bon français, le français des dimanches), mais surtout qu'il résiste aux incohérences, aux rangs desquelles se distingue la contradiction avec des faits de langue, même quand ceux-ci sont sous le feu d'un projecteur métalinguistique qui oblige à un accès à la conscience et à un savoir représenté. Confrontés à des imparfaits du type A midi l'avion s'écrasait, ou Tous les dimanches il allait faire du vélo, ou Il y avait un bon film ce soir à la télé, mais je ne pourrai pas le regarder, les Français interrogés, quasi unanimement, parlent de l'imparfait en termes de durée et de référence au passé. On a bien là un discours métalinguistique figé, où les termes n'ont plus grand sens commun, où la référenciation et l'adéquation à l'objet ne sont plus en cause. Les automatismes métalinguistiques de l'enseignement primaire et du collège sont prégnants. Les généralités métalinguistiques ont une vie autonome.

Il s'agit d'un fonctionnement à vide de microsystèmes de représentations qui rappelle la notion de "pensée aveugle" chez Condillac, c'est-à-dire une pensée sans représentation. Il n'y a dans l'esprit que la représentation de signes, mais pas la représentation de ce que ces signes signifient.

C'est l'expression d'une pensée qui ne serait que langage, et on est loin d'une construction de notions possiblement opératoires dans l'acquisition. La remise en cause des connaissances métalinguistiques est encore plus difficile manifestement que la construction de représentations métalinguistiques nouvelles. Mais c'est sans doute là une banalité cognitive.

Or c'est le métadiscours qui peut aider à construire les catégories et les généralisations qui vont accompagner et soutenir l'activité métacognitive : on voit l'importance du choix de métatermes et de métadiscours adéquats et signifiants._

\subsection{En résumé...}

En résumé, et pour le plaisir d'une certaine causticité, on peut dire que, d'un côté, bien souvent, les partisans d'un enseignement métalinguistique fournissent de mauvaises règles du produit aux apprenants, et donc ne leur fournissent guère d'aides, et que de l'autre côté, les adversaires d'une intervention métalinguistique s'appuient fréquemment sur un double mythe : d'une part le mythe du non discours, et d'autre part le mythe, battu en brèche par les observations du Projet E.S.F. par exemple (Perdue, 1993), ou, dans une certaine mesure, par les travaux sur les programmes d'immersion, 
qu'il suffirait de "communiquer", trois ou quatre heures par semaine, pour maîtriser un nouveau système linguistique.

Il n'y a, en effet, pas de corrélation simple entre l'explication métalinguistique enseignée et une représentation métalinguistique opératoire chez l'apprenant, tout comme il n'existe pas une corrélation simple entre une approche sans explication métalinguistique et l'absence de connaissance et d'activité métalinguistiques de la part de l'apprenant.

Il n'existe pas non plus de descriptions de l'intégration des processus conscients et non conscients, de la composition des activités épilinguistiques et métalinguistiques.

On ne peut guère en dire plus, et le "chemin des écoliers" est bien imprévisible, dans l'état actuel des connaissances, et au vu du nombre de paramètres tant cognitifs et affectifs que sociaux en jeu.

Mais dans toutes ces situations d'acquisition/apprentissage, s'exercent des activités langagières et métalangagières. S'exercent aussi des interférences, linguistiques et métalinguistiques, avec le système préalable de la langue maternelle et les représentations que les sujets ont sur elle.

L'"input" linguistique auquel les sujets ont été, et sont, exposés est un facteur qu'il est très rare de pouvoir cerner, autrement que dans des expériences très particulières.

L'"input" métalinguistique reçu par enseignement est plus facilement analysable, mais l'"input" métalinguistique spontané, l'"auto-input", issu de l'activité métalinguistique des sujets et de leurs métadiscours intérieurs ne l'est pas.

\section{Pour conclure...}

Fort heureusement, des représentations métalinguistiques parcellaires, voire fausses, n'entravent en rien le fonctionnement "réel" de la langue maternelle, ni même apparemment la compréhension en langue étrangère (Trévise, 1994), mais assurément en partie la production en langue étrangère quand elle est "réfléchie", et échappe aux imitations spontanées et automatiques, et aux hypothèses non conscientes. Les mouvements cognitifs d'automatisation ne se font heureusement pas, du moins en compréhension. On a donc intérêt à distinguer phénomènes de production et de reconnaissance. Peut-être l'activité métalinguistique est-elle plus prégnante en production qu'en compréhension, où l'activité épilinguistique serait plus sollicitée. La symétrie n'existe manifestement pas. C'est une autre "composition" complexe que l'on ne sait expliquer : celle des activités de compréhension et de production et de leurs liens en acquisition. Il s'agirait là aussi de faire la part entre activités conscientes et non conscientes dans cette composition, que ce soit à l'écrit ou à l'oral, sans oublier les 
interactions entre activités écrites et orales quand l'acquisition concerne les deux domaines.

Question triviale : pourquoi un enfant acquiert-il jusqu'à la perfection ce qu'un étranger a tant de mal à apprendre, et qu'un scientifique sait si insuffisamment (encore) percevoir et décrire, ou décrire et percevoir?

Une des raisons pour lesquelles l'étranger adulte arrive si rarement à la perfection est qu'il "possède" (est possédé par ?) un système préalable par lequel sa représentation du monde, de lui-même et du langage, et donc son identité, notamment inconsciente, affective et sociale, se sont forgées. L'étrangeté de la langue "étrangère" sera le plus souvent irréductible, non pas tant dans ses règles que dans ses modes de représentation préférentiels, ou ses jeux de mots, ses homophonies et polyphonies particulières. La langue étrangère n'aura jamais le statut de la langue "maternelle", celle qui a grandi avec nous, et avec laquelle nous avons grandi.

En ce qui concerne le scientifique, linguiste, psycholinguiste ou sociolinguiste, une des difficultés est peut-être qu'il ne peut procéder comme le fait l'enfant qui, dans sa construction simultanément cognitive et linguistique non consciente, se construit des catégories opérationnelles, fonctionne par analogies, et ne peut se permettre d'avoir des catégories figées qui le rendraient aveugle à l'observation et à la prise en compte de la réalité des fonctionnements linguistiques, qu'il s'agisse d'une langue 1 ou d'une langue 2. Il est vrai que l'enfant prend part à une énorme quantité d'échanges linguistiques dans des interactions où s'élabore son rapport identitaire à lui-même et aux autres. Le scientifique, plus obéissant des traditions grammaticales, moins actif aussi, s'enferme trop souvent dans des métadiscours trop catégorisés, trop rigides, ou au contraire des métaphores mal contrôlées, qui ne peuvent rendre compte à la fois de ce qui est stable et général, mais aussi du dynamisme du foisonnement langagier dans les usages particuliers, du jeu herméneutique. Il éprouve beaucoup de difficultés à y intégrer suffisamment une théorie du sujet humain qui habite ce langage non transparent et en est habité.

Une théorie du sujet implique la prise en compte de l'inconscient et de l'idéologie, et de l'assujettissement du sujet à ce non-contrôlable. On voit à quel point tenter de mesurer le rôle de la conscience ne peut être qu'une petite partie de l'étude de l'activité langagière. Mais on peut néanmoins avoir conscience de cette violence incontrôlable, comme ce bilingue, interviewé par Deprez, qui reconnaissait :

Je parle italien avec des Italiens, je commence la phrase en italien et je la termine en français, s'il-vous-plaît ! Si ! C'est fou, c'est complètement fou, ça vient tout seul (...) et $c^{\prime}$ est parti pour le français ${ }^{4} . .$.

\footnotetext{
4 (communication personnelle) souligné par moi.
} 


\section{Références bibliographiques}

ARRIVE, M. (1994). Langage et psychanalyse, linguistique et inconscient. Paris : P.U.F.

AUROUX, S. (1996). La philosophie du langage. Paris : P.U.F.

AUTHIER-REVUZ, J. (1995). Ces mots qui ne vont pas de soi. Boucles réflexives et non-coïncidences du dire. Paris : Larousse.

BENVENISTE, E. (1966). Problèmes de linguistique générale, tome 1. Paris : Gallimard.

BENVENISTE, E. (1974). Problèmes de linguistique générale, tome 2. Paris : Gallimard.

BESSE, H. et PORQUIER, R. (1991). Grammaires et didactiques des langues. Paris : Hatier-CREDIF..

BORRILLO, A. (1985). "Discours ou métadiscours ?", D.R.L.A.V., 32 : 47-61.

BREMER, K., ROBERTS, C., VASSEUR, M.-T., SIMONOT, M. et BROEDER, P. (1996). Achieving Understanding. London : Longman

BRESSON, F. (1991). "Le développement cognitif et l'apprentissage des langues", in C. Luc (ed.), Les langues vivantes à l'école élémentaire, Paris : I.N.R.P. : 21-26.

CULIOLI, A. (1967). "La communication verbale, l'aventure humaine", Encyclopédie des sciences de l'homme, Vol. IV, L'Homme et les autres, Paris : Editions Grange Batelière : 65-73.

CULIOLI, A. (1973). "Sur quelques considérations en linguistique", Communications, $20: 83-91$.

CULIOLI, A. (1974). "A propos des énoncés exclamatifs", Langue Française 22 : 6-16.

CULIOLI, A. (1990). Pour une linguistique de l'énonciation. Tome 1, Paris : Ophrys.

DANON-BOILEAU, L. (1994). "Le méta comme faculté de détour", Cahiers d'Acquisition et de Pathologie du Langage, 12, Paris : C.N.R.S.-Université R. Descartes : 71-77.

DEPREZ, C. (1994). Les enfants bilingues : langues et familles. Paris : Didier.

DESCLES, J.-P. (1994). "Réflexions sur les grammaires cognitives". Modèles Linguistiques, XV, 1. : 69-98.

DE VOGÜE, S. (1989). "Discret, dense, compact : les enjeux énonciatifs d'une typologie lexicale, in J.-J. Franckel (ed.), La notion de prédicat, Collection E.R.A., Université Paris 7 : 1-37.

ELLIS, R. (1990). Instructed Second Language Learning. Oxford, Blackwell.

ELLIS, R. et RATHBONE, M. (1986). The Acquisition of German in a classroom Context : an initial Report on the Ab-initio Acquisition of German in a College of Higher Education, mimeograph, Ealing College of Higher Education.

FRANCKEL, J.-J. et PAILLARD, D. (1992). "Objet : construction et spécification d'occurrences", Le Gré des Langues, 4, Paris : Editions L'Harmattan : 29-43.

FRANÇOIS, F. (1994). "Métalangage, folie, interprétation. Quelques remarques sur Perceval le Fou, autobiographie d'un schizophrène", Cahiers d'Acquisition et de Pathologie du Langage, 12, Paris : C.N.R.S.-Université R. Descartes : 99-123.

GRIZE, J.-B. (1990). Logique et Langage. Paris : Ophrys.

GRIZE, J.-B.(1996). Logique naturelle et communications. Paris : P.U.F. 
GÜLICH, E. (1994). "Commentaires métadiscursifs et "mise en scène" de l'élaboration du discours", Cahiers d'Acquisition et de Pathologie du Langage, 12, Paris : C.N.R.S.-Université R. Descartes : 29-51.

JAKOBSON, R. (1963). Essais de linguistique générale. Paris : Editions de Minuit, réédité Gallimard, Points, 1970.

KELLERMANN, E. (1980). "Oeil pour oeil", Encrages, Université de Paris 8 : 54-63.

KLEIN, W. (1986). Second Language Acquisition. Cambridge : C.U.P.

LAVER, J. (1973) "The detection and correction of slips of the tongue", in V. Fromkin (ed.), Speech Errors as Linguistic Evidence, La Haye : Mouton : 1-16.

LECERCLE, J.-J. (1990). The Violence of Language. Londres : Routledge.

MOREL, M.-A. (1985). "Etude de quelques réalisations de la fonction métadiscursive dans un corpus d'échanges oraux", D.R.L.A.V., 32 : 93-116.

PERDUE, C. (1993), (ed.). Adult Language Acquisition. Crosslinguistic Perspectives. 2 vol. Cambridge : C.U.P.

REY-DEBOVE, J. (1978). Le métalangage. Paris : Editions Le Robert.

REY-DEBOVE, J. (1985). "Le métalangage en perspective", D.R.L.A.V., 32 : 21-32.

ROBINSON, P. (1996). "Learning simple and complex second language rules under implicit incidental rule search and instructed conditions", S.S.L.A., $18: 27-67$.

SCHLYTER, S. (1995), (ed.). L'acquisition bilingue simultanée préscolaire, A.I.L.E., 6.

TREVISE, A. (1979) : "Spécificité de l'énonciation didactique dans l'apprentissage de l'anglais par des étudiants francophones", Encrages, in C. Perdue \& R. Porquier (eds), Linguistique appliquée, Encrages, Saint-Denis : Université de Paris VIII : 44-52.

TREVISE, A. (1984), en collaboration avec C. DEPREZ-DE HEREDIA. "Les malentendus, effets de loupe sur certains phénomènes d'acquisition d'une langue étrangère", in C. Noyau \& R. Porquier (eds), Communiquer dans la langue de l'autre, Saint-Denis : Presses Universitaires de Vincennes : 130-152.

TREVISE, A. (1992). "La gestion cognitive de l'étrangeté dans l'acquisition d'une langue étrangère", A.I.L.E.,1: 87-106.

TREVISE, A (1993). "Acquisition/apprentissage/enseignement d'une langue 2 : modes d'observation, modes d'intervention", Etudes de Linguistique Appliquée, 92 : 3650 .

TREVISE, A. (1994). "Invariance et spécificités dans l'acquisition d'une langue dite "étrangère" : rôle de l'activité métalinguistique", Cahiers d'Acquisition et de Pathologie du Langage, 12, Paris : C.N.R.S.-Université R. Descartes : 53- 69.

TREVISE, A. (1995). "She smoked a cigarette : elle fumait une cigarette ou elle fuma une cigarette ? Prétérit simple et construction de la télicité", S.I.G.M.A., 17-18, Université de Provence : 9-36.

TREVISE, A. (à paraître-a). "Contrastive metalinguistic representations: the case of "very French" learners of English", in C. James (ed.), Language Awareness, Special Issue, Contrastive perspectives on Language Awareness.

TREVISE, A. (à paraître-b). "Métalexique, métadiscours et interactions métalinguistiques", in C. Tisset (ed.), Actes du colloque des 2 et 3 octobre 1996. Groupe de recherches Jan Comenius, Université de Paris X-Nanterre.

WIDDOWSON, H. G. (1990). Aspects of Language Teaching. Oxford : O.U.P. 